Reply

\title{
Reply to "Overstated Claims of Efficacy and Safety. Comment On: Optimal Nutritional Status for a Well-Functioning Immune System Is an Important Factor to Protect against Viral Infections. Nutrients 2020, 12, 1181"
}

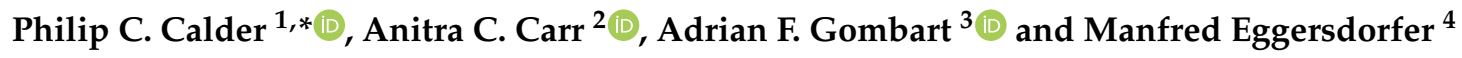 \\ 1 Faculty of Medicine, University of Southampton and NIHR Southampton Biomedical Research Centre, \\ University Hospital Southampton NHS Foundation Trust, Southampton SO16 6YD, UK \\ 2 Nutrition in Medicine Research Group, Department of Pathology and Biomedical Science, University of Otago, \\ Christchurch 8140, New Zealand; anitra.carr@otago.ac.nz \\ 3 Linus Pauling Institute, Department of Biochemistry and Biophysics, Oregon State University, \\ Corvallis, OR 97331, USA; adrian.gombart@oregonstate.edu \\ 4 Department Internal Medicine, University Medical Center Groningen, 9713 GZ Groningen, The Netherlands; \\ m.eggersdorfer@bluewin.ch \\ * Correspondence: pcc@soton.ac.uk
}

Received: 25 August 2020; Accepted: 27 August 2020; Published: 3 September 2020

check for updates

We thank Vorland et al. [1] for their interest in our recent publication [2]. They provide comments in relation to our interpretation of systematic reviews of omega-3 (n-3) fatty acids [3] and vitamin C [4-6]. The systematic review of $n-3$ fatty acids, sometimes in combination with other nutrients, in acute respiratory distress syndrome (ARDS) was cited in the context of a so-called "cytokine storm" rather than protection from viral, or any other, infection per se. The findings from that systematic review are shown in Table 1.

Table 1. Summary of the findings of the systematic review and meta-analysis of the effects of n-3 fatty acid rich formulas in patients with acute respiratory distress syndrome [3].

\begin{tabular}{cccc}
\hline Outcome & Effect & 95\% CI & $p$ \\
\hline $\mathrm{PaO}_{2} / \mathrm{FiO}_{2}$ at day 4 (Mean difference mmHg) & 38.88 & $10.75-67.02$ & 0.0068 \\
\hline $\mathrm{PaO}_{2} / \mathrm{FiO}_{2}$ at day 8 (Mean difference mmHg) & 23.44 & $1.73-45.15$ & 0.034 \\
\hline Ventilator days (Mean difference d) & -2.24 & $-3.77-0.71$ & 0.0042 \\
\hline New organ failure (Relative risk) & 0.45 & $0.32-0.63$ & $<0.00001$ \\
\hline Length of intensive care unit stay (Mean difference d) & -3.09 & $-5.19-0.99$ & 0.004 \\
\hline 28 day mortality (Relative risk) & 0.64 & $0.49-0.84$ & 0.0015 \\
\hline All cause mortality (Relative risk) & 0.79 & $0.59-1.07$ & Not given \\
\hline
\end{tabular}

CI, confidence interval.

In our view, these data support our statement that the systematic review identifies "a significant improvement in blood oxygenation and significant reductions in ventilation requirement, new organ failures, length of stay in the intensive care unit and mortality at 28 days". In our publication [2] we made a recommendation for intake of eicosapentaenoic acid plus docosahexaenoic acid of $250 \mathrm{mg} /$ day, which is in keeping with many recommendations made by regulatory authorities for these fatty acids [7-9] and can be easily achieved through diet (i.e., consumption of fatty fish) [10]. It is important 
to emphasise that the amount of $n-3$ fatty acids delivered in formulas used in the trials in ARDS is greatly in excess of $250 \mathrm{mg} /$ day, typically several g per day.

Our statements in relation to the systematic review on vitamin C and pneumonia [4] were that there was "a significant reduction in the risk of pneumonia with vitamin $C$ supplementation, particularly in individuals with low dietary intakes" and that "in older patients, disease severity and risk of death were reduced with supplementation, particularly in the case where initial plasma levels of vitamin C were low". Thus, we stress the likely role of vitamin C in those individuals with low intake or status. This is consistent with the findings reported in the systematic review, the abstract of which makes the final conclusion "therapeutic vitamin C supplementation may be reasonable for pneumonia patients who have low vitamin $C$ plasma levels because its cost and risks are low". In relation to the systematic review on vitamin $C$ and the common cold [5] we stated that "vitamin $C$ supplementation has also been shown to decrease the duration and severity of upper respiratory tract infections, such as the common cold, and significantly decrease the risk of infection when given prophylactically in people under enhanced physical stress". The results section of the abstract of the systematic review states "in adults the duration of colds was reduced by $8 \%$ (3\% to $12 \%)$ and in children by $14 \%(7 \%$ to $21 \%)$. In children, 1 to $2 \mathrm{~g}$ /day vitamin $\mathrm{C}$ shortened colds by $18 \%$. The severity of colds was also reduced by regular vitamin C administration". Furthermore, the conclusions section of the abstract of the systematic review states "vitamin C may be useful for people exposed to brief periods of severe physical exercise. Regular supplementation trials have shown that vitamin $C$ reduces the duration of colds, but this was not replicated in the few therapeutic trials that have been carried out. Nevertheless, given the consistent effect of vitamin $C$ on the duration and severity of colds in the regular supplementation studies, and the low cost and safety, it may be worthwhile for common cold patients to test on an individual basis whether therapeutic vitamin C is beneficial for them". We note that risk ratio for the incidence of common colds in those undertaking heavy acute physical activity was 0.48 ( $95 \%$ confidence interval 0.35-0.64). We consider that our statements are consistent with the findings of these two systematic reviews.

Vorland et al. [1] raise the important question of safety. We do not dispute that safety is of utmost importance, and in accordance with this, we state that "intakes should follow recommended upper safety limits set by expert authorities, such as the European Food Safety Authority and, in the United States, the IOM" and we go on to discuss intakes of vitamins C and D in relation to stated tolerable upper limits and where these vary in children.

Our review deals with micronutrients (and $n-3$ fatty acids), viral infection and related respiratory disease; it is not restricted solely to COVID-19. There are not yet any published trials of supplementation with individual or mixed micronutrients and either prevention or treatment of COVID-19; we wish to emphasise that we did not give any impression that such evidence currently exists. However, given that there is a substantial body of evidence that micronutrients support the function of many components of the immune system [11] and may reduce risk of incidence or severity of respiratory illness $[2,11]$, we consider that such trials are warranted.

Author Contributions: Conceptualization, P.C.C.; writing-original draft preparation, P.C.C.; writing-review and editing, A.C.C., A.F.G., M.E. All authors have read and agreed to the published version of the manuscript.

Funding: This research received no external funding.

Conflicts of Interest: P.C.C. has research funding from BASF AS and Bayer Consumer Care; acts as an advisor/consultant to BASF AS, DSM, Danone/Nutricia, Cargill, Smartfish, Nutrileads, Bayer Consumer Care, and Pfizer (now GSK) Consumer Healthcare; has received reimbursement for travel and/or speaking from Danone, Fresenius Kabi, Baxter, Pfizer (now GSK) Consumer Healthcare, Abbott, Smartfish, Biogredia and the California Walnut Commission; and is President and member of the Board of Directors of the European Branch of the International Life Sciences Institute. A.C.C. has received research funding from Bayer Consumer Care and travel reimbursement from DSM. A.F.G. has received research funding from Bayer Consumer Care and has acted as an advisor/consultant for and has received reimbursement for travel and/or speaking from Bayer Consumer Care. M.E. acts as an advisor to DSM and has received travel reimbursement from DSM. He is member of the Scientific Board of PM International and President of the Gesellschaft für angewandte Vitaminforschung. 


\section{References}

1. Vorland, C.J.; Bohan Brown, M.M.; Kyle, T.K.; Brown, A.W. Overstated claims of efficacy and safety: Comments on: "Optimal nutrient status for a well-functioning immune system is an important factor to protect against viral infections". Nutrients 2020, 12, 1181. Nutrients 2020, 12, 2690.

2. Calder, P.C.; Carr, A.C.; Gombart, A.F.; Eggersdorfer, M. Optimal nutritional status for a well-functioning immune system is an important factor to protect against viral infections. Nutrients 2020, 12, 1181. [CrossRef] [PubMed]

3. Dushianthan, A.; Cusack, R.; Burgess, V.A.; Grocott, M.P.; Calder, P.C. Immunonutrition for acute respiratory distress syndrome (ARDS) in adults. Cochrane Database Syst. Rev. 2019, 1, CD012041. [CrossRef] [PubMed]

4. Hemilä, H.; Louhiala, P. Vitamin C for preventing and treating pneumonia. Cochrane Database Syst. Rev. 2013, 8, CD005532. [CrossRef] [PubMed]

5. Hemilä, H.; Chalker, E. Vitamin C for preventing and treating the common cold. Cochrane Database Syst. Rev. 2013, 1, CD000980. [CrossRef] [PubMed]

6. Ran, L.; Zhao, W.; Wang, J.; Wang, H.; Zhao, Y.; Tseng, Y.; Bu, H. Extra dose of vitamin C based on a daily supplementation shortens the common cold: A meta-analysis of 9 randomized controlled trials. BioMed Res. Int. 2018, 2018, 1837634. [CrossRef] [PubMed]

7. Food and Agricultural Organization. Fats and fatty acids in human nutrition-Report of an Expert Consultation. In FAO Food and Nutrition Paper; Food and Agricultural Organization: Rome, Italy, 2010.

8. Chinese Nutrition Society. Chinese Dietary Reference Intakes Summary; People's Medical Publishing House: Beijing, China, 2013; p. 16.

9. European Food Safety Authority. Scientific opinion on dietary reference values for fats, including saturated fatty acids, polyunsaturated fatty acids, monounsaturated fatty acids, trans fatty acids and cholesterol. EFSA J. 2010, 8, 1461.

10. Calder, P.C. Very long-chain n-3 fatty acids and human health: Fact, fiction and the future. Proc. Nutr. Soc. 2018, 77, 52-72. [CrossRef] [PubMed]

11. Gombart, A.F.; Pierre, A.; Maggini, S. A review of micronutrients and the immune system-working in harmony to reduce the risk of infection. Nutrients 2020, 12, 236. [CrossRef] [PubMed] 